\title{
Molecular Dynamics of Natural Rubber/Layered Silicate Nanocomposites as Studied by Dielectric Relaxation Spectroscopy
}

\author{
Marianella Hernández ${ }^{1,2^{*}}$, Tiberio A. Ezquerra ${ }^{3}$, Javier Carretero-González ${ }^{2}$, Raquel \\ Verdejo $^{2}$, Miguel A. López-Manchado ${ }^{2 *}$ \\ ${ }^{1}$ Universidad Simón Bolívar, Departamento de Mecánica, Valle de Sartenejas, Caracas 1081, Venezuela. \\ ${ }^{2}$ Instituto de Ciencia y Tecnología de Polímeros, CSIC, Madrid 28006, España. \\ ${ }^{3}$ Instituto de Estructura de la Materia, CSIC, Madrid 28006, España. \\ *corresponding author: marherna@usb.ve/marherna@ictp.csic.es or 1manchado@ictp.csic.es
}

\begin{abstract}
The local and global motions of polymer chains in non vulcanized and vulcanized natural rubber/layered silicate nanocomposites were studied by dielectric relaxation spectroscopy, with special consideration to the effect of clay type and loading on the time and length scale of the relaxation processes. Results reveal that the type and concentration of clay do not have an effect on the segmental mode of the NR matrix, while the vulcanization reaction shifts this relaxation to lower frequencies. In addition, a new slower mode attributable to restricted segmental dynamics is found in the nanocomposites where a highly intercalated structure is achieved, independently of having the rubber matrix vulcanized or not. The study of this new slower segmental relaxation contributes to a better understanding of the dynamics of rubber/layered silicate nanocomposites, with the intention of improving our knowledge on the reinforcement effects manifested in this kind of composites with low filler loadings.
\end{abstract}

\section{Introduction}

Nanotechnology is recognized as one of the most promising fields of research of $21^{\text {st }}$ century. The beginning of nanotechnology and nanoscience research can be traced back over 40 years. However, it was in the past decade that the world witnessed bigger strides of this technology from both academic and industrial points of view ${ }^{1-4}$.

The term "nanocomposite" refers to every type of composite material having fillers in the nanometer size range, at least in one dimension. For such nanocomposites the total interfacial phase becomes the critical parameter, rather than the volume fraction of the filler ${ }^{5-7}$. Because of their nanofillers dispersion, nanocomposites exhibit markedly improved mechanical, thermal, electrical and gas barrier properties, when compared to pure polymers or their traditional composites. The most common nanosized fillers are inorganic clay minerals consisting of nanolayered silicates. The stacking of the layers of approximately $1 \mathrm{~nm}$ thickness by weak dipolar forces leads to interlayers or galleries between the layers. These galleries are occupied by cations such as $\mathrm{Na}^{+}$and $\mathrm{Ca}^{++}$which can be replaced by organic cations such as alkyl ammonium cations via ion exchange reaction rendering to obtain hydrophobic surfaces. This ion exchange produces organophilic clays called organoclays which are more compatible with the polymer ${ }^{8,9}$.

The literature search shows that several research groups have prepared nanocomposites based on plastics and rubbers with nanoclays. In particular, rubber/layered silicate nanocomposites are increasingly attracting scientific and technological attention, because of the high reinforcing efficiency of the nanosilicate, even at very load loading $(<10 \mathrm{wt} \%)^{10-14}$. The high specific surface area is believed to be one of the reasons why the nature of reinforcement is different in composites based on nanofillers. It is expected to provide enhanced interphase effects and tensile strength. Nevertheless, there is yet no 
satisfactory theoretical explanation for the origin of improvement of the properties of polymer nanocomposites ${ }^{15}, 16$. Results obtained by various experimental techniques, as well as by theory and computer simulations, indicate the presence of an interfacial polymer layer around the filler, with structure/morphology and chain dynamics modified with respect to the bulk polymer matrix ${ }^{7,16-18}$.

Broadband dielectric spectroscopy is a powerful tool for the investigation of molecular dynamics of polymers and composites. Dielectric property analysis of filled polymers contributes to a better understanding of the structure-property relationships at the morphological level. More specifically, in order to relate the macroscopic properties of nanocomposites with molecular concepts, one must understand the molecular motions or dynamics of these materials in response to various applied fields.

When placed in an electric field, nanocomposites are subjected to ionic, interfacial, and dipole polarization. These polarization mechanisms have considerable different time and length scales, making dielectric spectroscopy, uniquely suited for the study of nanocomposite dynamics ${ }^{19}$. Therefore, motional processes which take place for polymeric systems on extremely different time scales can be investigated in a broad frequency and temperature range. Such motional processes can be localized fluctuations within a backbone segment or local rotational fluctuations of a short side chain. On a larger spatial and longer time scale and for temperatures above the glass transition the so called segmental motion or $\alpha$-relaxation becomes relevant. At more extended length scale, for polymers with a component of the dipole moment parallel to the chain, a further process can be observed called normal-mode relaxation, in which the translational motion of the whole chain characterized by the end-to-end vector takes place. In this case, the molecular dipole vectors that are parallel to the repeating unit are summed up over the chain, and therefore the normal-mode relaxation is related to both the geometry and the dynamics of the macromolecule ${ }^{20-22}$.

Several authors have extensively studied the dynamics of 1,4-cis-poly(isoprene) (PI) by using dielectric spectroscopy ${ }^{19}, 23-26$. Due to the lack of symmetry in its chemical structure PI has nonzero components of the dipole moment both perpendicular and parallel to the chain contour. Thus, two dielectric relaxation processes, the segmentaland the normal- mode process, are present. Moreover, Mijovic et al ${ }^{19}$ have studied nanocomposites of organically modified clay nanoparticles and a PI matrix. Nonetheless, no in-depth study of the dielectric properties of natural rubber (NR) nanocomposites has been undertaken, analyzing the relaxation spectra and correlating the molecular motion of chains with its structure. Furthermore, to our best knowledge there are not yet extensive experimental studies on NR nanocomposites relaxation spectra and its relationship with the composition, structure and properties of these materials.

Therefore, the aim of this paper is to study the effect of the presence of the filler on the local and global motions of polymer chains in NR/nanolayered silicate composites. At first, we report results from dielectric spectroscopy, examining how the segmental and normal modes of these polymer chains are affected by the presence of silicate particles. In addition, we show the effect of the concentration of clay on the time and length scale of the relaxation processes, plus a comparison between nanosilicates. We also discuss on the influence of the vulcanization and how the crosslinking process affects the dielectric spectra of the nanocomposites.

\section{Experimental Section}


2.1. Materials. Natural rubber (NR) was kindly supplied by Malaysian Rubber (Berhad, Malaysia) under the trade name CV 60 (Mooney viscosity: $\operatorname{ML}(1+4) 100{ }^{\circ} \mathrm{C}=60$ ). Two layered silicates were employed in this study, in particular, a natural sodiummontmorillonite described as Cloisite ${ }^{\circledR} \mathrm{Na}^{+}(\mathrm{CNa}+)$ and this montmorillonite modified with a quaternary ammonium salt, Cloisite ${ }^{\circledR} 15 \mathrm{~A}$ (C15A). Both samples were provided by Southern Clay Products Inc (Gonzales, USA). The structural characteristics of the clays are reported in Table 1.

Table 1. Technical characteristics of the layered silicates employed in this work

\begin{tabular}{lccc}
\hline Clay & $\begin{array}{c}\text { Organic } \\
\text { modifier }\end{array}$ & $\begin{array}{c}\text { Modifier concentration } \\
\text { (mequiv/100g clay) }\end{array}$ & $\begin{array}{c}\text { Basal spacing, } d \\
(\AA)\end{array}$ \\
\hline Cloisite ${ }^{\circledR} \mathrm{Na}^{+}(\mathrm{CNa}+)$ & none & --- & 11.7 \\
Cloisite ${ }^{\circledR} 15 \mathrm{~A}(\mathrm{C} 15 \mathrm{~A})$ & $2 \mathrm{M} 2 \mathrm{HT}^{\mathrm{a}}$ & 125 & 31.5 \\
\hline
\end{tabular}

a 2M2HT: dimethyl, dehydrogenated tallow, quaternary ammonium. The tallow consists in $\sim 65 \%$ of $\mathrm{C}_{18}, \sim 30 \%$ of $\mathrm{C}_{16}$, and $\sim 5 \%$ of $\mathrm{C}_{14}$, and a chloride anion ${ }^{27}$.

2.2. Preparation of NR nanocomposites. Non vulcanized nanocomposites were prepared consisting of the pure gum and the layered silicate $(1,2.5,5,7.5$ and $10 \mathrm{phr}$ (parts per hundred of rubber)). The samples are referred as $\mathrm{NR} / x y$, where $x$ and $y$ correspond to the amount and type of clay respectively. With respect to the vulcanized samples, the formulation of natural rubber compounds expressed as (phr) is as follows: sulphur (2.5), zinc oxide (5), stearic acid (1), MBTS (benzothyazyl disulfide) (1), antioxidant PBN (phenyl $\beta$-naphthylamine) (1), and layered silicate (5 and 10). The nanocomposite samples were prepared in an open two-roll laboratory mill at room temperature. The rotors operated at a speed ratio of 1:1.4. Rubber compounds were vulcanized at $150{ }^{\circ} \mathrm{C}$ in an electrically heated hydraulic press using the optimum cure time ( $\mathrm{t}_{90}$ ) derived from the curing curves previously determined by means of a Rubber Process Analyzer (RPA2000 Alpha Technologies). These samples are referred as vulcNR/xy, following the same nomenclature as for the non vulcanizates. Identical methodology was applied to both layered silicates nanocomposites (NR/C15A and $\mathrm{NR} / \mathrm{CNa}+$ ), thus affording a quantitative comparison of their dynamics.

2.3. Characterization. Broad-band dielectric relaxation spectroscopy (DRS) measurements were performed on an ALPHA high resolution dielectric analyzer (Novocontrol Technologies GmbH, Hundsangen, Germany). Film samples prepared from the nanocomposites were mounted in the dielectric cell between two parallel goldplated electrodes. The complex permittivity $\varepsilon^{*}$ of a given sample can be calculated from the measurement of the complex impedance $Z^{*}$ given by

$$
Z *(\omega)=\frac{U *(\omega)}{I *(\omega)}
$$

Where $U^{*}$ and $I^{*}$ are the voltage and current circulating through the sample at a certain angular frequency $\omega$. Once the impedance has been measured, $\varepsilon^{*}$ can be calculated by means of

$$
\varepsilon^{*}(\omega)=\varepsilon^{\prime}-i \varepsilon^{\prime \prime}=\frac{1}{i \omega Z^{*}(\omega) C_{0}}
$$

Where $\varepsilon^{\prime}$ and $\varepsilon^{\prime \prime}$ are the real and imaginary part of the complex permittivity and $C_{0}$ corresponds to the capacity of the empty sample holder. The complex permittivity of the nanocomposites was measured over a frequency window of $10^{-1}<F / \mathrm{Hz}<10^{7}$ ( F= $\omega / 2 \pi$ is the frequency of the applied electric field) in the temperature range from -150 to $150{ }^{\circ} \mathrm{C}$ in $5^{\circ} \mathrm{C}$ steps. 
The imaginary part of the obtained dielectric permittivity was analyzed by the phenomenological Havriliak-Negami $(\mathrm{HN})$ function including a conductivity term ${ }^{20,28}$

$$
\varepsilon^{*}(\omega)=\varepsilon_{\infty}+\frac{\Delta \varepsilon}{\left[1+\left(i \omega \tau_{\mathrm{HN}}\right)^{b}\right]^{c}}-i \frac{\sigma}{\varepsilon_{\mathrm{vac}} \omega^{s}}
$$

Where $\Delta \varepsilon=\varepsilon_{s}-\varepsilon_{\infty}, \varepsilon_{\infty}$ and $\varepsilon_{s}$ are the unrelaxed and relaxed values of the dielectric constant and $\tau_{\mathrm{HN}}$ is a characteristic relaxation time. In eq. $3, b$ and $c$ are shape parameters $(0<b, c \leq 1)$ which describe the symmetric and the asymmetric broadening of the equivalent relaxation time distribution function, respectively. Here, $\sigma$ is related to the direct current electrical conductivity, $\varepsilon_{\mathrm{vac}}$ is the vacuum dielectric constant, and $s$ depends on the nature of the conduction mechanism.

Differential scanning calorimetry (DSC) measurements (Perkin-Elmer DSC7) in the temperature range from $-75^{\circ} \mathrm{C}$ to $75^{\circ} \mathrm{C}$ were also employed to investigate thermal transitions.

\section{Results and Discussion}

3.1 Broad-band dielectric relaxation spectroscopy (DRS) of NR nanocomposites. Figure 1 describes the dielectric loss spectra for neat NR and its nanocomposites over a wide range of frequency at different temperatures. In all the spectra, the loss peaks are shifting to higher frequencies with increasing temperature. This shift in the frequency of the maxima is characteristic of thermal activated processes.

Similarly to synthetic 1,4 -cis-poly(isoprene) $(\mathrm{PI})^{19,23,24,26}$, two relaxation modes are present in the NR (Figure 1a). For temperatures above the glass transition temperature $\left(T_{g}=-64{ }^{\circ} \mathrm{C}\right)$ the segmental mode related to the segmental motions of the polymer chain is observed. At higher temperatures, a more intense process which can be assigned to the normal mode is clearly detected. In both nanocomposites (Figures $1 \mathrm{~b}$ and 1c) the segmental mode, or $\alpha$-relaxation, is still detectable and appears located in the same temperature range as for neat NR. A second process appears at higher temperatures in the NR/5C15A compound (Figure 1b) which apparently differs from the normal mode of neat NR. We will refer to this process hereafter as "new mode" that will be further discussed. This new mode seems to be absent in the NR/5CNa+ nanocomposite (Figure 1c) which only exhibits the segmental mode and an additional process at low temperatures most likely due to the electric dipole rotations of the absorbed water in the silicate gallery ${ }^{15}, 29$. Moreover, at high temperatures conductivity dominates the low frequency tail of the spectrum of the $\mathrm{NR} / 5 \mathrm{CNa}+$ compound, thus no defined relaxation process can be appreciated.

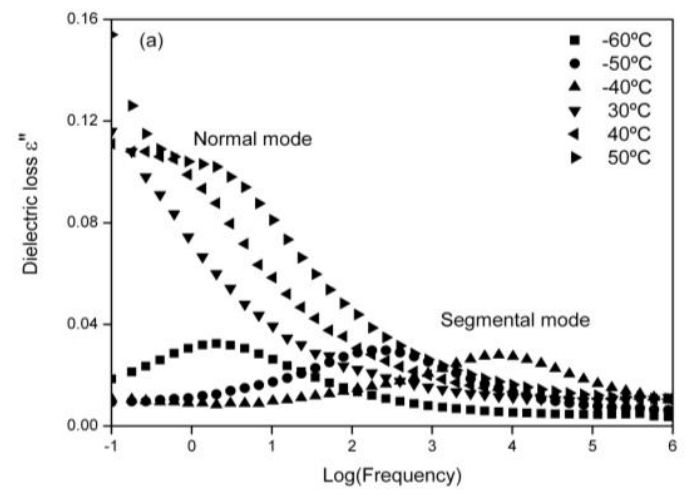



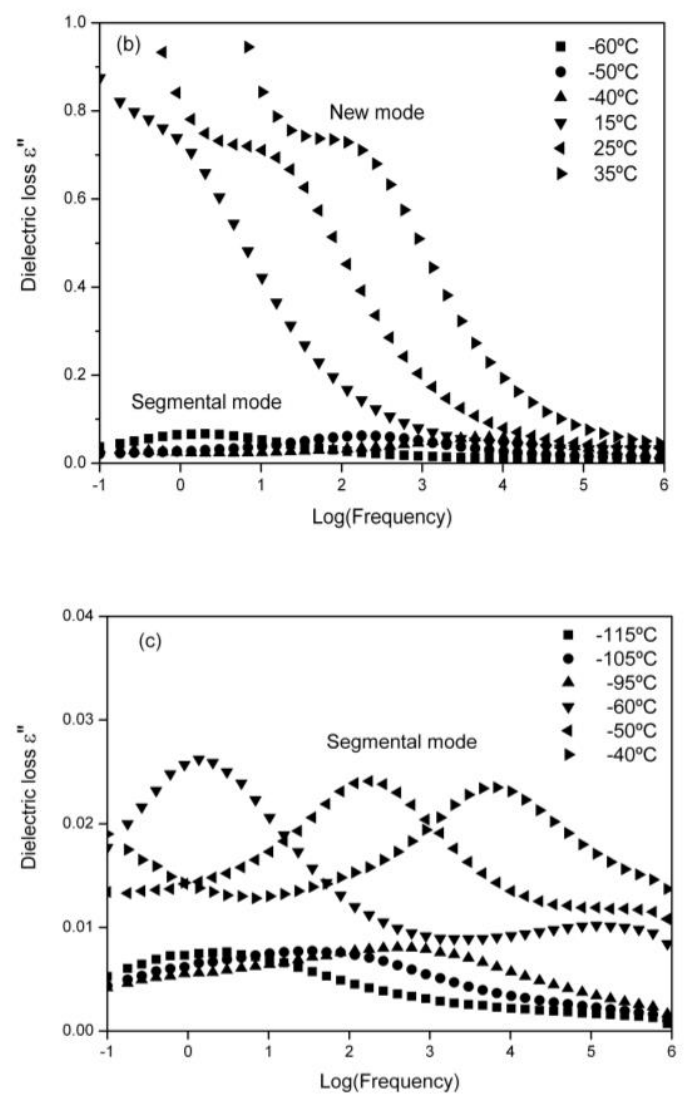

Figure 1. Dielectric loss $\varepsilon^{\prime \prime}$ vs. frequency for: (a) Neat NR; (b) NR/5C15A nanocomposite; and (c) $\mathrm{NR} / 5 \mathrm{CNa}+$ nanocomposite.

In order to better examine the different relaxations present in all compounds, the discussion of our results will be divided into low temperature and high temperature processes.

3.1.1 Low temperature process: segmental relaxation. Figure 2 shows selected dielectric loss spectra at $\mathrm{T}=-50{ }^{\circ} \mathrm{C}$ for $\mathrm{NR}$ and the $\mathrm{NR} / 5 \mathrm{C} 15 \mathrm{~A}$ and $\mathrm{NR} / 5 \mathrm{CNa}+$ nanocomposites. From the spectra hereby presented, we can notice that the segmental mode process ( $\alpha$-relaxation) is well resolved in the frequency domain and shows a relatively broad and asymmetric peak regardless the type of filler incorporated in the nanocomposite if compared to the spectrum of neat NR. These results suggest the independence of the glass transition process with the silicate incorporated. Besides, the invariance of the glass transition has been verified when the silicate type and content varies from 1 to $10 \mathrm{phr}$. Thus, no significant changes on the segmental molecular dynamics of the NR come into sight by the addition of layered nanosilicates in the bulk state. 


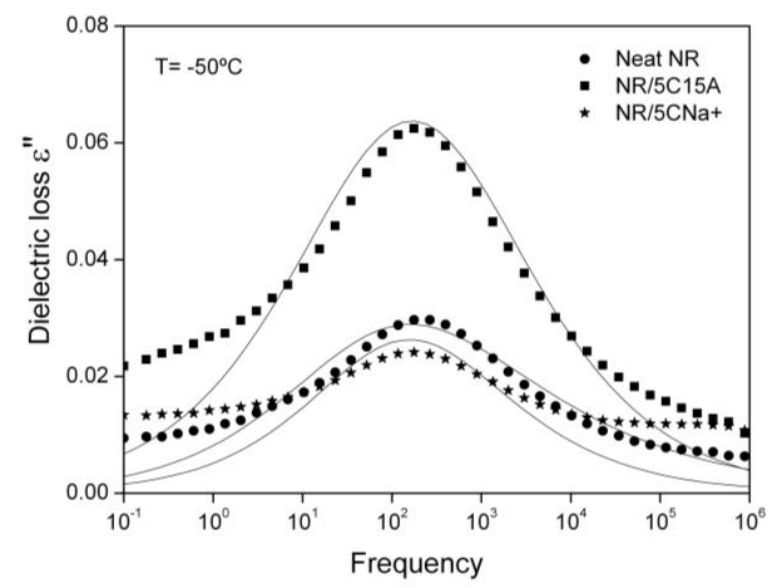

Figure 2. Frequency dependence of dielectric loss $\varepsilon^{\prime \prime}$ of the NR nanocomposites indicated on the plot in the region of the segmental mode. Solid lines correspond to HN fitting.

One can also see that all three spectra can be well described by the HN function. The characteristic parameters of this function (see eq.3) such as $\Delta \varepsilon, b, c, \tau_{\mathrm{HN}} \sigma$ and $s$ were obtained. Table 2 shows these parameters for temperatures in the region of the segmental mode.

Table 2. HN parameters of segmental mode of NR and its nanocomposites.

\begin{tabular}{cccccccc}
\hline Compound & $T, \mathrm{~K}$ & $\Delta \varepsilon$ & $b$ & $c$ & $\tau_{\mathrm{HN}}(\mathrm{s})$ & $\sigma(\mathrm{S} / \mathrm{cm})$ & $s$ \\
\hline \multirow{3}{*}{ Neat NR } & 218 & 0.143 & 0.720 & 0.359 & $2.490^{*} 10^{-2}$ & $1.0^{*} 10^{-20}$ & 0.36 \\
& 223 & 0.158 & 0.505 & 0.617 & $2.037 * 10^{-3}$ & $1.0^{*} 10^{-20}$ & 0.31 \\
& 228 & 0.151 & 0.448 & 0.869 & $1.666^{*} 10^{-4}$ & $1.0 * 10^{-20}$ & 0.30 \\
NR/5C15A & 218 & 0.295 & 0.686 & 0.395 & $2.651^{*} 10^{-2}$ & $1.0^{*} 10^{-20}$ & 0.25 \\
& 223 & 0.305 & 0.545 & 0.578 & $2.291^{*} 10^{-3}$ & $1.0 * 10^{-20}$ & 0.24 \\
& 228 & 0.301 & 0.458 & 0.888 & $1.626^{*} 10^{-4}$ & $1.0^{*} 10^{-20}$ & 0.23 \\
NR/5CNa+ & 218 & 0.129 & 0.633 & 0.380 & $3.065^{*} 10^{-2}$ & $8.5 * 10^{-19}$ & 0.38 \\
& 223 & 0.121 & 0.451 & 0.874 & $9.055^{*} 10^{-3}$ & $1.0^{*} 10^{-20}$ & 0.16 \\
\hline
\end{tabular}

It is seen from Table 2 that the shape parameters $b$ and $c$ show systematic deviation. With increasing temperature, $b$ decreases slightly whereas the $c$ parameter rises to higher values. This behaviour is observed for all samples under study.

We corroborated that the position of the segmental mode was independent of silicate type and content by analyzing the temperature dependence of the relaxation time $\left(\tau_{\max }\right)$ obtained from the $\mathrm{HN}$ fitting parameters

$$
\tau_{\max }=\frac{1}{2 \pi F_{\max }}=\tau_{\mathrm{HN}}\left[\sin \frac{b \pi}{2+2 c}\right]^{-1 / b}\left[\sin \frac{b c \pi}{2+2 c}\right]^{1 / b}
$$

The corresponding values for $\tau_{\max }$, shown in Figure 3 , reveal the Vogel-FulcherTamman (VFT) dependence of $\tau_{\max }$ with the reciprocal temperature as

$$
\tau_{\max }=\tau_{0} \exp \left(\frac{B}{T-T_{0}}\right)
$$

Where $\tau_{0}$ and $B$ are empirical parameters, and $T_{0}$ is the so-called ideal glass transition or Vogel temperature, which is generally $30-70 \mathrm{~K}$ below $T_{g}{ }^{21}$. To reduce the effect of data 
fitting to the VFT equation over a limited frequency range, a value of $\log _{10} \tau_{0} \approx 14$ was assumed according to previous work ${ }^{30}$.

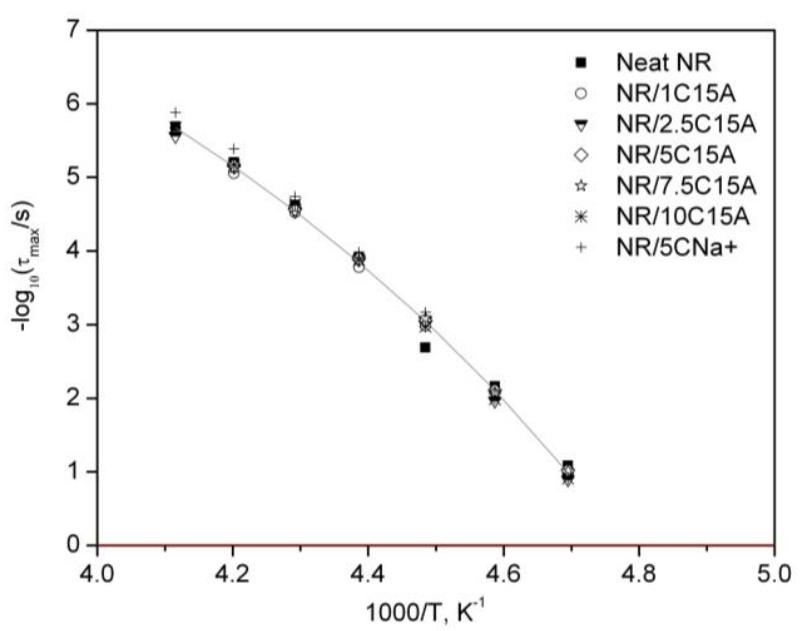

Figure 3. Temperature dependence of the average relaxation time for the segmental mode of NR and its nanocomposites with clay loading as a parameter.

In Figure 3 we can clearly see that neither an increase in nanosilicate loading nor the type of filler added has an effect on the time scale of the segmental mode process of the NR matrix.

3.1.2 High temperature processes: new and normal modes. Figure 4 a shows selected dielectric loss spectra at $\mathrm{T}=40{ }^{\circ} \mathrm{C}$ for $\mathrm{NR}$ and the $\mathrm{NR} / 5 \mathrm{C} 15 \mathrm{~A}$ and $\mathrm{NR} / 5 \mathrm{CNa}+$ nanocomposites in the temperature region where the "new mode" is observed for the $\mathrm{NR} / 5 \mathrm{C} 15 \mathrm{~A}$. From the spectra presented in this figure, we observe that neither NR nor $\mathrm{NR} / 5 \mathrm{CNa}+$ exhibit this new mode, being only detectable for the nanocomposite with the additive $\mathrm{C} 15 \mathrm{~A}$. The nanoclay $\mathrm{C} 15 \mathrm{~A}$ is a montmorillonite modified with a quaternary ammonium salt. This modification results in a more pronounced intercalation of the NR chains, as corroborated by previous studies ${ }^{12,14,15}$. We could expect a restricted mobility of the polymer chains close to the polymer/filler interface due to an enhanced interaction of the NR chains with the clay surface. These interactions should not be present in the unfilled NR matrix or conventional NR composites with poorer intercalation as in those based on unmodified clay $(\mathrm{CNa}+)$. 

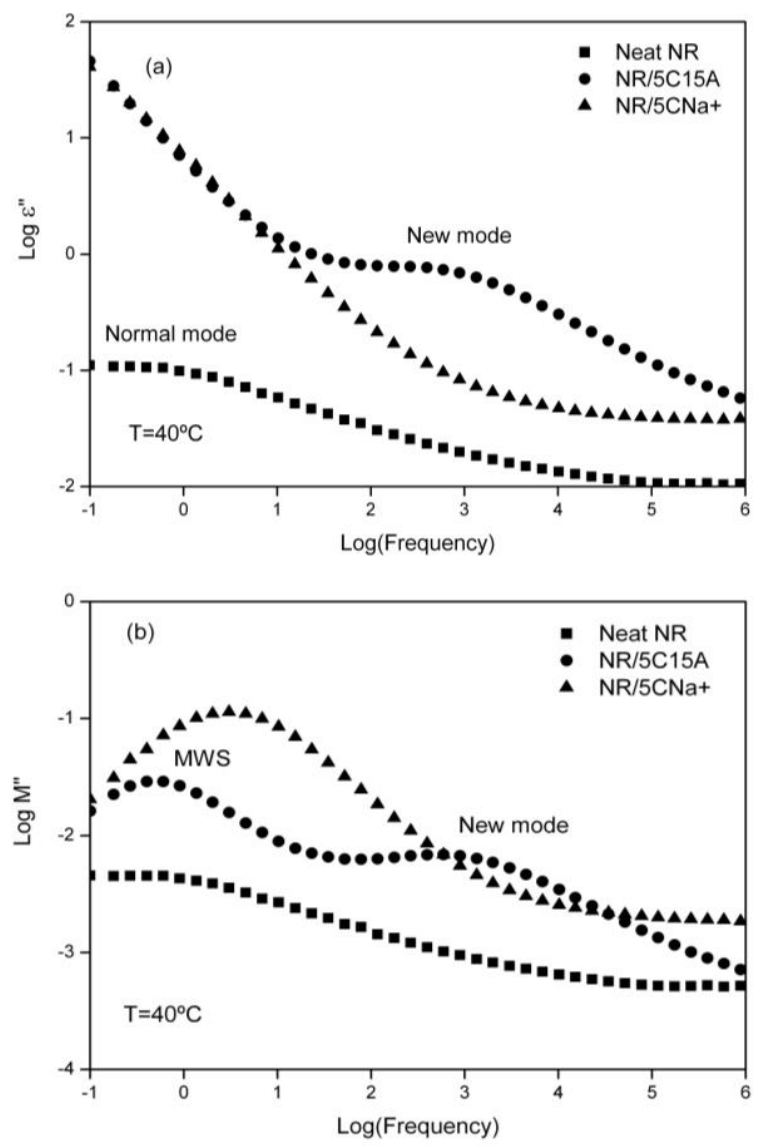

Figure 4. (a) Dielectric loss $\varepsilon^{\prime \prime}$, and (b) Dielectric loss modulus $M^{\prime \prime}$, in the frequency domain for neat NR and its nanocomposites.

In order to better understand the physical nature of the new mode, the dielectric modulus formalism was employed (see Figure $4 \mathrm{~b}$ ). The permittivity and modulus formalisms describe the same electrical relaxation phenomena; however, under different conditions a specific formalism could allow to extract more information with respect to the occurring physical processes. In this study, the recorded dielectric data were initially expressed in terms of real and imaginary part of permittivity and then transformed, via eq 6 , to the electric modulus formalism:

$$
M^{*}=\frac{1}{\varepsilon^{*}}=\frac{1}{\varepsilon^{\prime}-i \varepsilon^{\prime \prime}}=\frac{\varepsilon^{\prime}}{\varepsilon^{\prime 2}+\varepsilon^{\prime 2}}+i \frac{\varepsilon^{\prime \prime}}{\varepsilon^{\prime 2}+\varepsilon^{\prime 2}}=M^{\prime}+i M^{\prime \prime}
$$

Where $M^{\prime}$ is the real and $M^{\prime \prime}$ the imaginary part of the electric modulus, respectively. In particular, the interpretation of relaxation phenomena via the electric modulus formalism offers some advantages upon other treatments, since large variations in the permittivity and loss at low frequencies and high temperatures are minimized. Further, difficulties occurring from the electrode nature, the electrode-specimen contact and the injection of space charges and absorbed impurities can be neglected. Arguments and examples for the resulting benefits of the electric modulus presentation have been presented elsewhere ${ }^{31,32}$.

The modulus spectra of nanocomposites offer an added insight into their dynamics because high conductivity makes identification of relaxation processes in the permittivity spectra difficult, though not impossible ${ }^{33}$. Figure $4 \mathrm{~b}$ shows the dielectric modulus spectra of the pure gum, NR, and its nanocomposites (NR/5C15A and $\mathrm{NR} / 5 \mathrm{CNa}+$ ). In the low frequency region, a relaxation process can be observed in the 
nanocomposites regardless the type of clay added, while this process is absent in the pure NR. The incorporation of nanoclay caused additional relaxation dispersion due to an interfacial polarization at the nanoclay/polymer interface, the so-called MaxwellWagner-Sillars (MWS) effect. Interfacial polarization is always present in materials comprised of more than one phase. This kind of polarization arising at the interfaces is due to the migration of charge carriers through different phases of the composite material resulting in differential charge accumulation at the interfaces. When these charges are made to move by the application of an external electric field, the motion will be hindered at various points of the composite material differently, causing space charge to appear. The appearance of such space charge can distort the macroscopic field and appears as polarization to an external observer ${ }^{34}$.

Figure $4 \mathrm{~b}$ also reveals for the NR/5C15A nanocomposite the presence of the "new mode" reflected in Figure 4a accompanied at lower frequencies by the MWS interfacial polarization.

Some interesting features can be noted regarding the influence of clay content on the "new mode" by representing the dielectric strength as a function of the filler content (Figure 5) for the NR/C15A nanocomposites. Here we can notice a slight increasing tendency in dielectric strength $(\Delta \varepsilon)$ with clay loading. This behaviour could induce us to assign the "new mode" to a restricted segmental mobility phase of the polymer close to the polymer/filler interface.

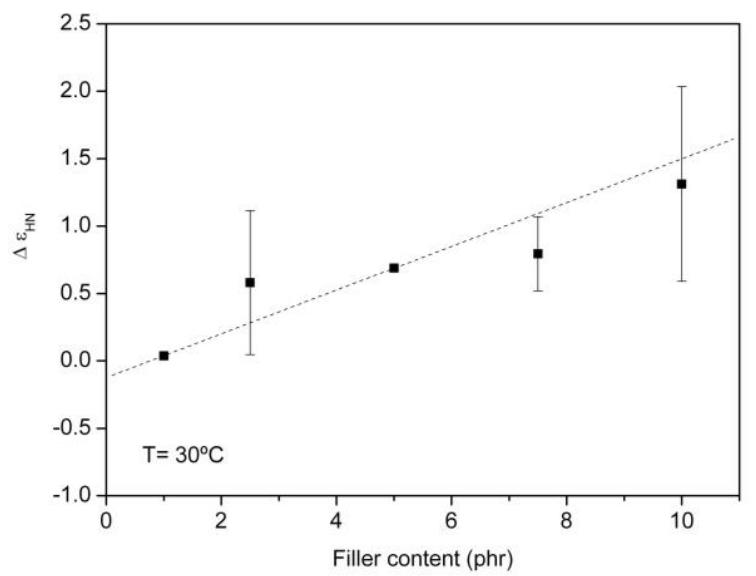

Figure 5. Relaxation strength as a function of clay loading for the NR/C15A nanocomposites.

In addition, the data presented in Figures 6 and 7 reveal that this new relaxation becomes faster with increasing filler content, levelling of for loadings higher than $5 \mathrm{phr}$. In principle one would expect confinement effects to be increasingly important as filler content increases. In general, segmental dynamics may become faster by confinement when the confinement volume becomes comparable to that of the cooperative rearranging regions ${ }^{35}$.

In our case, the fastening of the "new mode" dynamics with increasing filler content can be understood as due to a reduction of volume available for the polymer chains intercalated within the galleries formed by the silicate layers. This would imply that an increasing number of layered silicates accommodate NR chains in the central area of the intergallery without expanding the basal spacing, thus resulting in increased relaxation rate $^{19,31,36}$. Interestingly, however, when higher content of clay is added (up to $10 \mathrm{phr}$ ) one could presume that a percolated network of nanoparticles that can influence relaxation could be formed resulting in restriction effects. Nevertheless, this issue awaits more detailed and systematic study. 


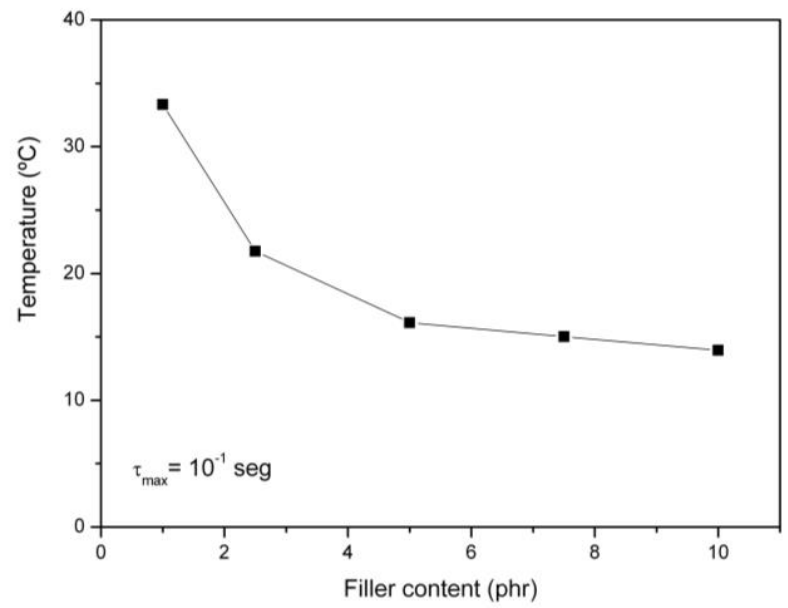

Figure 6. Temperature dependence of the "new mode" at $\tau_{\max }=10^{-1} \mathrm{~s}$ for NR/C $15 \mathrm{~A}$ nanocomposites with clay loading.

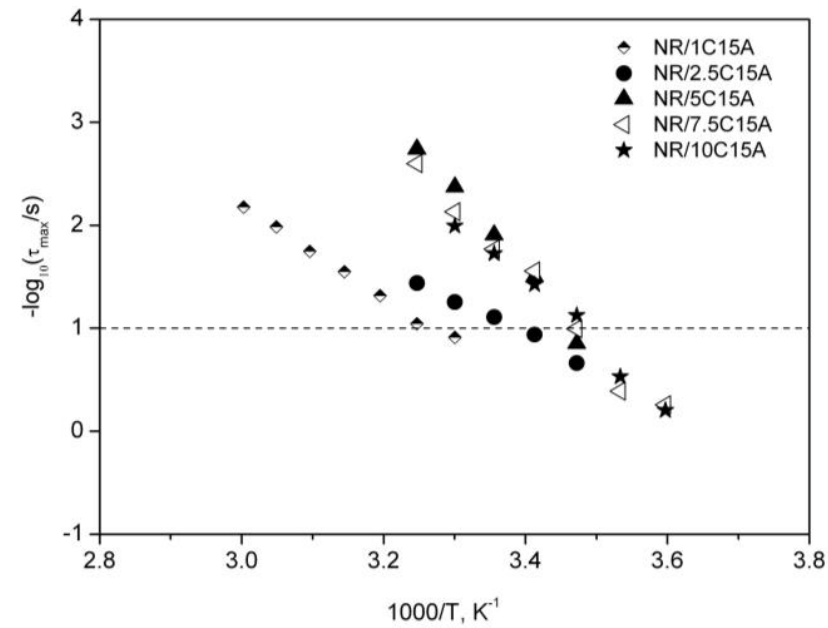

Figure 7. Temperature dependence of the average relaxation time for the "new mode" of NR/C $15 \mathrm{~A}$ nanocomposites with clay loading as a parameter.

With the intention of further characterize the "new mode" present in the NR/C15A nanocomposites, DSC measurements were analyzed. Figure 8 shows DSC heating thermograms of neat NR and four nanocomposites with the clay content (in phr) indicated on the plot. A single glass transition around $-70^{\circ} \mathrm{C}$ is observed for all samples. This evidences that the thermodynamic glass transition of NR/C15A nanocomposites corresponds to the NR matrix, in agreement with the dielectric data shown in Figure 2 for the segmental mode. At first glance, there is no evidence of a second thermodynamic $T_{\mathrm{g}}$ which could be directly related to the "new mode". However, it has been recently demonstrated using computer simulations that a continuous distribution of relaxation times as we approach a solid interface can lead to a double loss peak in the susceptibility, and this has been proposed as an alternative interpretation for this kind of response $\mathrm{e}^{17,37,38}$. 


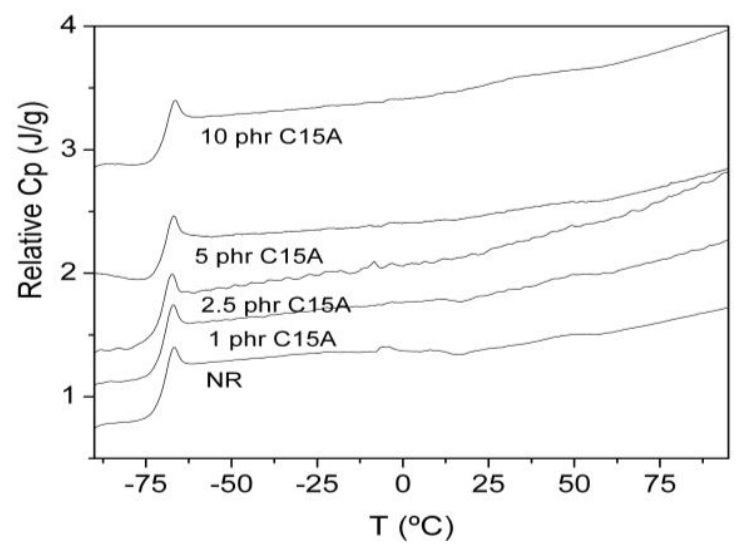

Figure 8. DSC heating thermograms (second runs, displaced vertically for clarity) obtained for the NR/C15A nanocomposites with the samples indicated on the plot.

Normal mode was examined next. If we analyze the spectrum of neat NR (Figure 1a) we can clearly see that the normal mode process is present at high temperatures at frequencies below the $\alpha$-process and overlapped with the electrical conductivity contribution. The overall chain dynamics is reflected by this process.

A careful analysis of the VFT temperature dependence of the high temperature processes (Figure 9) evidences that the global chain dynamics (normal mode) of NR is slower than the segmental mode and exhibits weaker temperature dependence than that of the $\alpha$-relaxation. This can be understood by considering that the segmental motion proves smaller length scales than those proven by the normal mode process ${ }^{20}$. In between the normal and the segmental modes of the NR matrix, lays the "new mode" of the $\mathrm{NR} / 5 \mathrm{C} 15 \mathrm{~A}$ nanocomposite, which is clearly slower than the segmental mode, but faster than the normal mode process of NR.

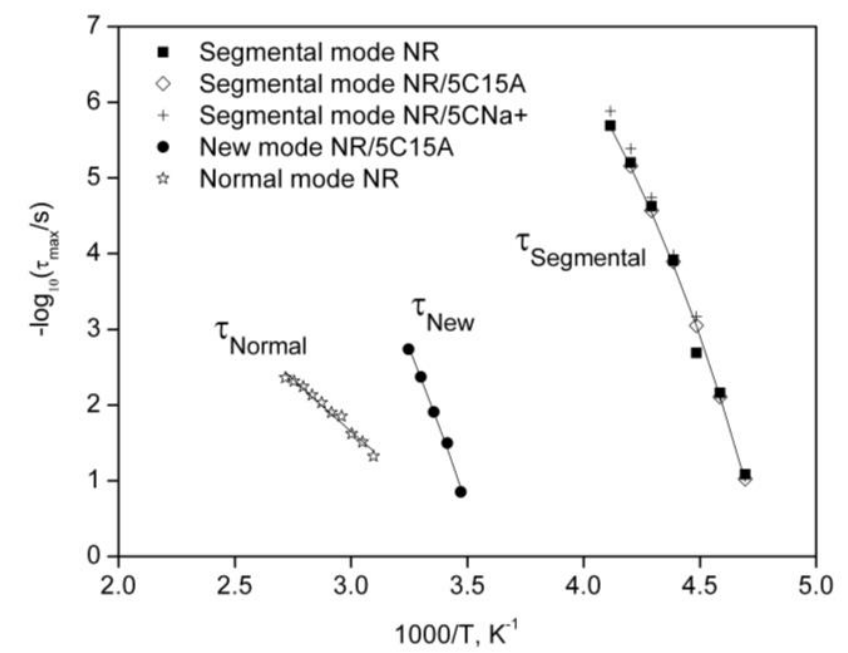

Figure 9. Temperature dependence of the average relaxation time for normal, new and segmental mode of neat NR and its nanocomposites. Solid lines are fits to the VFT functional form.

At this point, in order to deeper analyze the nature of the "new mode" present in the NR/C15A nanocomposites; we evaluated the vulcanized NR/silicate nanocomposites.

3.2 Vulcanized NR nanocomposites. The dielectric measurements have been proven to be useful for studying vulcanization of various kinds of rubber since the nature of relaxation phenomena for polymers depends on various factors including crosslinking density. The most common method of vulcanizing rubber is to mix it with sulphur and 
heat it up to $100-150{ }^{\circ} \mathrm{C}$. This procedure results in crosslinking of the polymer chains by mono- or polysulphide bridges. A side reaction is also known to proceed along crosslinking, leading to formation of heterocyclic groups in the polymer chains. In sulphur vulcanizates correspondingly the following polar groups are found: (a) the carbonyl groups present in the unvulcanized rubber and those formed during vulcanization; (b) mono- or polysulphide crosslinks; (c) S-C bonds in the heterocyclic groups in the main chains ${ }^{39}$. In particular, in vulcanized NR, the change in the segmental mobility is generally observed as a shift of the glass-rubber transition to higher temperatures and an increase in the dispersion amplitudes ${ }^{39}$.

In the following sections, the relaxation phenomena present in sulphur vulcanized NR and in vulcanized NR/layered silicate nanocomposites, as illustrated in Figure 10, are discussed in the same way as with their non vulcanized equivalents, regarding the temperature range where the relaxation process appeared.
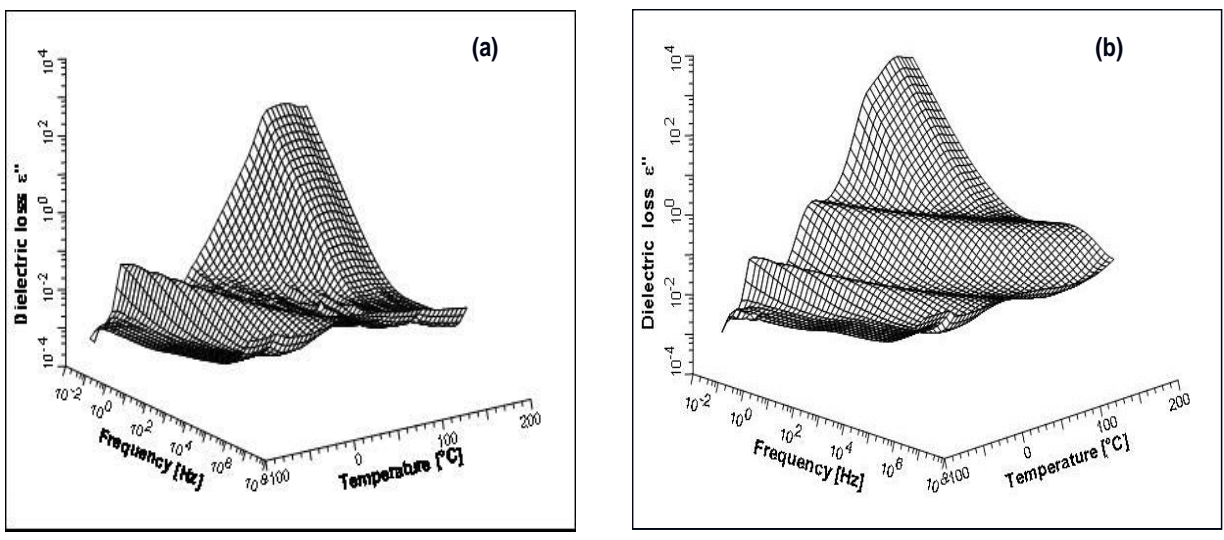

Figure 10. 3D representation of the frequency and temperature dependence of the dielectric loss $\varepsilon^{\prime \prime}$ for vulcanized samples of (a) NR and (b) NR/5C15A nanocomposite.

3.2.1 Effect of vulcanization on the segmental dynamics. For the sake of comparison, Figure 11 shows the dielectric loss data for the vulcanized samples of NR, NR/5C15A and $\mathrm{NR} / 5 \mathrm{CNa}+$ in the temperature region of the segmental dynamics. The corresponding spectrum for unvulcanized NR is also shown. The results indicate that vulcanizing by sulphur induces a slowing down of the segmental dynamics and a significant increase of the dispersion amplitudes. The later effect indicates that the side reaction resulting in heterocyclic groups is effective because they would hinder the segmental motion and they would increase the total number of polar groups involved, with a subsequent increase of the dispersion amplitude or the loss maximum ${ }^{39}$.

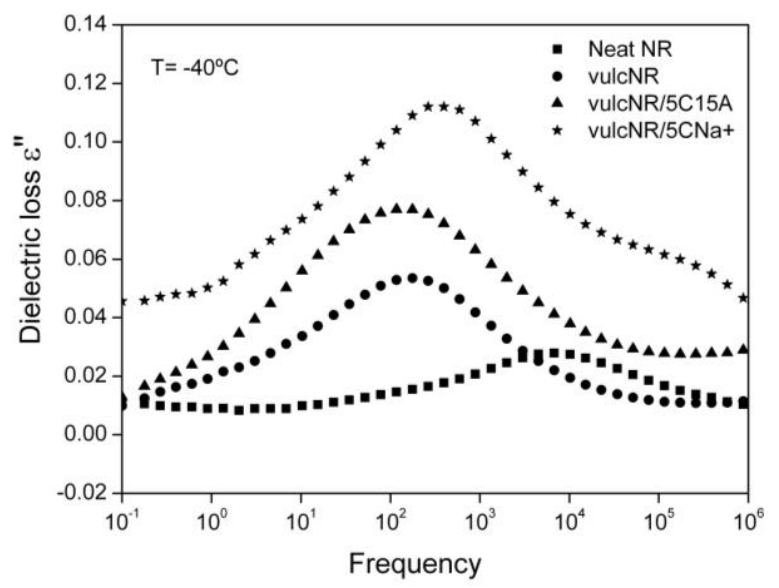


Figure 11. Frequency dependence of dielectric loss $\varepsilon^{\prime \prime}$ for vulcanized samples of NR and NR/5C15A and $\mathrm{NR} / 5 \mathrm{CNa}+$ nanocomposites in the temperature region of the segmental dynamics. The corresponding spectrum for neat NR is also shown.

Figure 12 shows the relaxation times of the segmental mode corresponding to vulcanized samples of $\mathrm{NR}, \mathrm{NR} / 5 \mathrm{C} 15 \mathrm{~A}, \mathrm{NR} / 10 \mathrm{C} 15 \mathrm{~A}$ and $\mathrm{NR} / 5 \mathrm{CNa}+$. The relaxation times for NR have been also included. The observed increase in the average relaxation time due to the vulcanization reaction can be interpreted straightforward considering the network formation. It is evident that the formation of polysulphide crosslinks and cyclic sulphide structures cause restrictions on the segmental motions of the polymer chains. Moreover, this high frequency process exhibits almost no dependence on the degree of filling ( 5 and $10 \mathrm{phr}$ of $\mathrm{C} 15 \mathrm{~A}$ ). The relaxation process for the vulcanized NR/5CNa+ nanocomposite, however, shows other tendency since the segmental mode seems to be faster if compared to the vulcanized NR/5C15A compound.

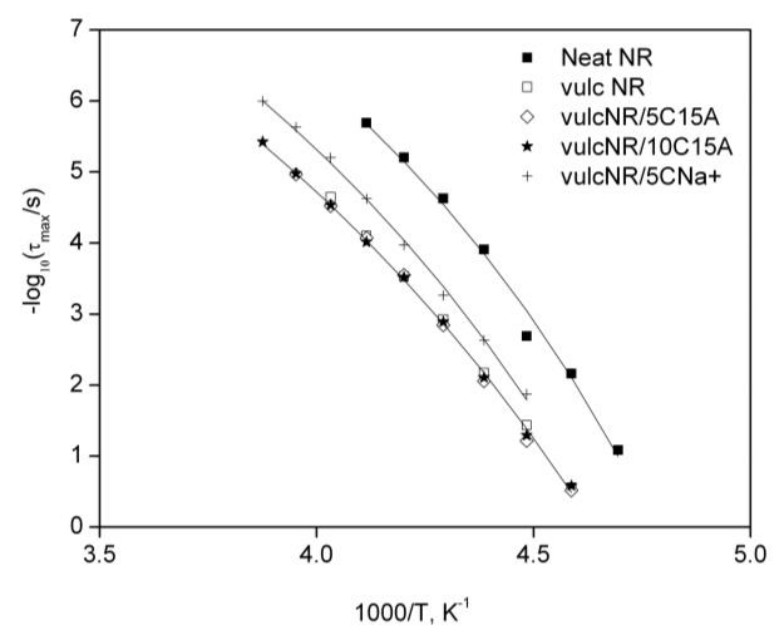

Figure 12. Temperature dependence of the average relaxation time of the segmental mode corresponding to vulcanized samples of NR and NR/C15A and NR/CNa+ nanocomposites.

3.2.2 Effect of vulcanization on the new and normal mode dynamics. For the sake of comparison, Figure 13a shows the dielectric loss data for the vulcanized samples of NR, $\mathrm{NR} / 5 \mathrm{C} 15 \mathrm{~A}$ and $\mathrm{NR} / 5 \mathrm{CNa}+$. The data corresponds to the temperature region where the normal mode of unvulcanized NR and the "new mode" of unvulcanized NR/5C15A are detected. It is clear the absence of a normal mode in the vulcanized samples, as seen in Figure 10. It is known that the normal mode of vulcanized NR becomes broader and decreases in amplitude upon vulcanization due to suppression of large-scale motions of the dipole oriented parallel to the polymer backbone ${ }^{20}$. However, the process corresponding to the "new mode" is present for the vulcanized NR/5C15A nanocomposite. Once again, we used the modulus formalism to better analyze this relaxation. 

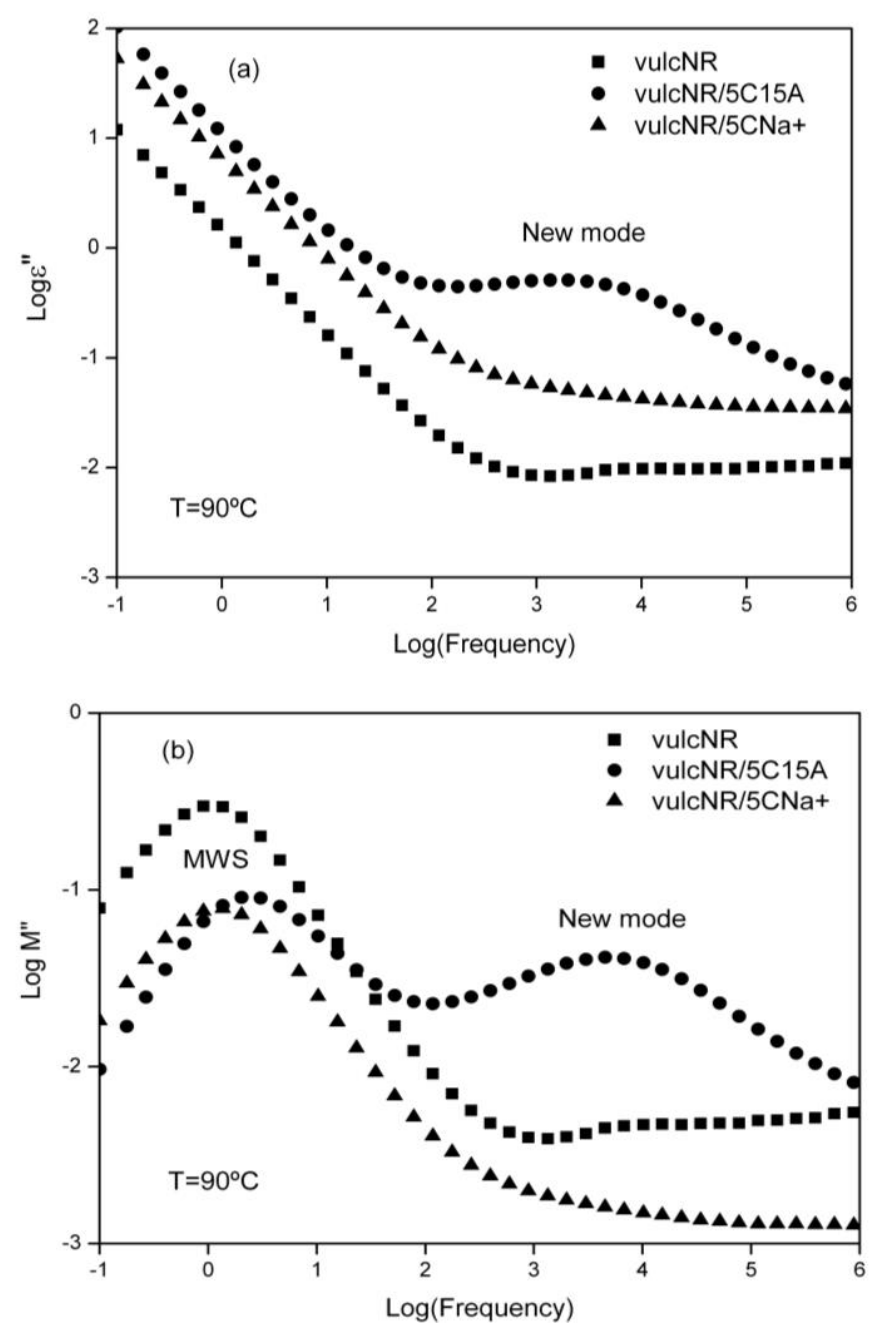

Figure 13. (a) Dielectric loss $\varepsilon^{\prime \prime}$, and (b) Dielectric loss modulus $M^{\prime \prime}$, in the frequency domain for vulcanized samples of $\mathrm{NR}$ and $\mathrm{NR} / 5 \mathrm{C} 15 \mathrm{~A}$ and $\mathrm{NR} / 5 \mathrm{CNa}+$ nanocomposites.

Figure $13 \mathrm{~b}$ illustrates the dielectric modulus spectra of vulcanized NR and its nanocomposites. The peak at low frequencies corresponds to the MWS relaxation. We believe that in vulcanized NR, this peak can be a consequence of the ingredients (sulphur, accelerators, activation complex, etc) added to the rubber matrix so the crosslinking reaction can take place. The addition of these components makes the polymeric material to be a heterogeneous media consisting of different phases with different dielectric permittivities and conductivities ${ }^{40-42}$. While for the vulcanized nanocomposites, the presence of the MWS peak has the same explanation as for the non vulcanized nanocomposites: the interfacial polarization (ion accumulation) that occurs at the interface between materials with differing dielectric constants, such as rubber and layered silicates.

At high frequencies the spectrum of the vulcanized NR/5C15A nanocomposite has similarities with the non vulcanized $\mathrm{NR} / 5 \mathrm{C} 15 \mathrm{~A}$ nanocomposite, since we note the presence of a second peak.

The temperature dependence of this new process is reflected in Figure 14, where we can clearly see that this "new mode" is associated to a restricted segmental dynamics. This slow down of the cooperative movements provides a clear evidence for the immobilization of polymer chains in the interface layer around the clay particles independently of having the rubber matrix vulcanized or not. We can notice that similar displacements of the curves have occurred in the segmental mode as well as in the new 
slower mode when the NR matrix is vulcanized. Thus, we can confirm the assumption drawn from the non vulcanized nanocomposites study; showing that the "new mode" present in the NR/C15A compounds corresponds effectively to a restricted segmental relaxation. We believe that part of the polymer chains are immobilized as an interface layer around the clay particles and so the interfacial effects dominate the bulk properties of the material.

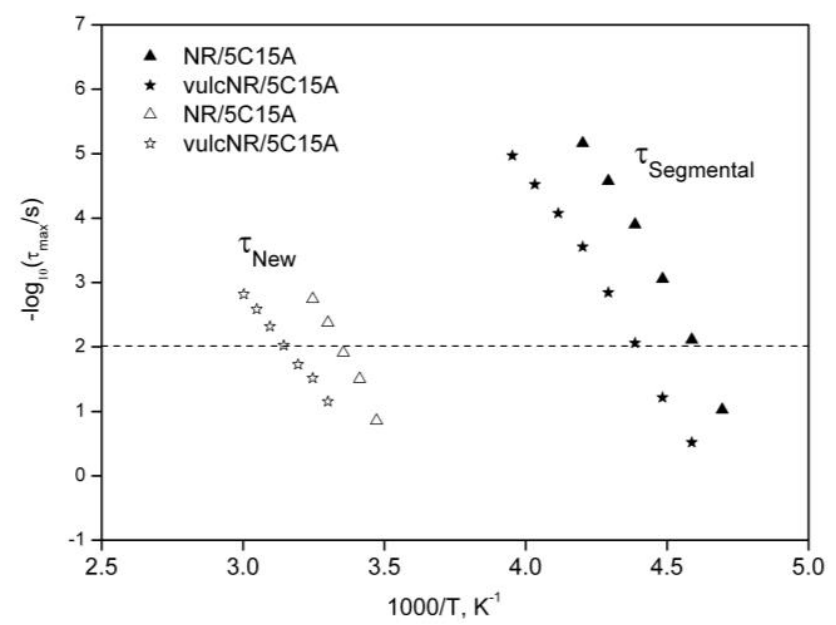

Figure 14. Temperature dependence of the average relaxation time for the segmental and "new" modes of non vulcanized and vulcanized samples of NR/5C15A nanocomposites.

\section{Conclusions}

Dielectric relaxation spectroscopy has been proven to be a powerful technique for investigating molecular dynamics in a series of nanocomposites consisting of NR and $\mathrm{C} 15 \mathrm{~A}$ and $\mathrm{CNa}+$ layered silicates. Different relaxation process have been detected and studied.

At low temperatures, the results for the molecular dynamics from dielectric measurements show that neither an increase in nanosilicate loading nor the type of filler added has an effect on the segmental mode process of the non vulcanized NR matrix. This segmental mode is related to segmental motions of the polymer chains.

At higher temperatures, the so-called Maxwell-Wagner-Sillars effect is present in all nanocomposites and is detected in the low frequency range, attributable to the interfacial polarization at the nanoclay/polymer interface. Besides, a new mode ascribed to a restricted segmental mode with a slower relaxation rate appears at higher frequencies in the NR/C15A nanocomposites attributed to the polymer chains under constraint because of the influence of the interfacial polymer-particle interactions, which modify the bulk polymer matrix dynamics. We have also observed that this restricted segmental mode is only detectable in nanocomposites where a highly intercalated structure is achieved, since the NR/CNa+ nanocomposites do not exhibit this relaxation. Concerning the effect of the vulcanization reaction on the NR and $\mathrm{NR} /$ silicate nanocomposites dynamics, at low temperatures the spectra obtained are characterized by a shift of the segmental mode towards lower frequencies. Vulcanizing with sulphur induces a slowing down of the segmental dynamics of the NR matrix. Moreover, this segmental process exhibits almost no dependence on the degree of silicate filling. Nonetheless, the type of clay added does have an influence on the time scale of the segmental dynamics since the NR/CNa+ nanocomposite.

While at high temperatures, the new relaxation process for the NR/C $15 \mathrm{~A}$ nanocomposites becomes visible as in the non vulcanized compounds, confirming that the nature of this mode is associated to restricted segmental dynamics. Finally, from our 
point of view, the study of this new slower segmental relaxation contributes to a better understanding of the structure/morphology relationship and polymer dynamics of layered silicate nanocomposites, with the intention of improving our knowledge on the reinforcement effects manifested in this kind of composites with low filler loadings.

Acknowledgements. The authors thank the financial support of the Spanish Ministry of Science and Innovation (MICCIN) through its project MAT 2007-61116. M. Hernández gratefully acknowledges the Mision Ciencia fellowship from the Venezuelan Ministry of Science and Technology and Dr. Alejandro Sanz for his help with the DSC measurements.

\section{References}

1. Winey, K. I.; Vaia, R. A. MRS Bull. 2007, 32, (4), 314-319.

2. $\quad$ Okada, A.; Usuki, A. Macromol. Mater. Eng. 2006, 291, (12), 1449-1476.

3. LeBaron, P. C.; Wang, Z.; Pinnavaia, T. J. Appl. Clay Sci. 1999, 15, (1-2), 1129.

4. Giannelis, E. P. Appl. Organomet. Chem. 1998, 12, (10-11), 675-680.

5. R. A. Vaia, H. D. W. Materials Today 2004, 6.

6. Ray, S.; Easteal, A. J. In Advances in polymer-filler composites: Macro to nano, International Composites Conference, Sydney, AUSTRALIA, Jul 11-14, 2006; Taylor \& Francis Inc: Sydney, AUSTRALIA, 2006; pp 741-749.

7. Schmidt, D.; Shah, D.; Giannelis, E. P. Curr. Opin. Solid State Mat. Sci. 2002, 6, (3), 205-212.

8. Viet, C. X.; Ismail, H.; Rashid, A. A.; Takeichi, T.; Thao, V. H. Polym.-Plast. Technol. Eng. 2008, 47, (11), 1090-1096.

9. Alexandre, M.; Dubois, P. Mater. Sci. Eng. R-Rep. 2000, 28, (1-2), 1-63.

10. Hakim, R. N.; Ismail, H. J. Reinf. Plast. Compos. 2009, 28, (12), 1417-1431.

11. Carretero-Gonzalez, J.; Valentin, J. L.; Arroyo, M.; Saalwachter, K.; LopezManchado, M. A. Eur. Polym. J. 2008, 44, (11), 3493-3500.

12. Avalos, F.; Ortiz, J. C.; Zitzumbo, R.; Lopez-Manchado, M. A.; Verdejo, R.; Arroyo, M. Eur. Polym. J. 2008, 44, (10), 3108-3115.

13. Lopez-Manchado, M. A.; Valentin, J. L.; Carretero, J.; Barroso, F.; Arroyo, M. Eur. Polym. J. 2007, 43, (10), 4143-4150.

14. Arroyo, M.; Lopez-Manchado, M. A.; Valentin, J. L.; Carretero, J. Compos. Sci. Technol. 2007, 67, (7-8), 1330-1339.

15. Carretero-Gonzalez, J.; Retsos, H.; Verdejo, R.; Toki, S.; Hsiao, B. S.; Giannelis, E. P.; Lopez-Manchado, M. A. Macromolecules 2008, 41, (18), 6763-6772.

16. Pissis, P.; Fragiadakis, D.; Kanapitsas, A.; Delides, K. In Broadband dielectric relaxation spectroscopy in polymer nanocomposites, 17th European Symposium on Polymer Spectroscopy (ESOPS 17), Seggauberg, AUSTRIA, Sep 09-12, 2007; Wiley-V C H Verlag Gmbh: Seggauberg, AUSTRIA, 2007; pp 12-20.

17. Fragiadakis, D.; Pissis, P.; Bokobza, L. Polymer 2005, 46, (16), 6001-6008.

18. Adnan, A.; Sun, C. T.; Mahfuz, H. Compos. Sci. Technol. 2007, 67, (3-4), 348356.

19. Mijovic, J.; Lee, H. K.; Kenny, J.; Mays, J. Macromolecules 2006, 39, (6), $2172-$ 2182.

20. Schönhals, F. K. A., Broadband Dielectric Spectroscopy. Springer: 2003.

21. Fitzgerald, J. P. R. a. J. J., Dielectric Spectroscopy of Polymeric Materials. Fundamentals and Applications. American Chemical Society: Washington, DC, 1997. 
22. Blythe, A. R., Electrical properties of polymers. Cambdrige University Press: Oxford, 1979.

23. Boese, D.; Kremer, F.; Fetters, L. J. Macromolecules 1990, 23, (6), 1826-1830.

24. Boese, D.; Kremer, F. Macromolecules 1990, 23, (3), 829-835.

25. Cerveny, S.; Zinck, P.; Terrier, M.; Arrese-Igor, S.; Alegria, A.; Colmenero, J. Macromolecules 2008, 41, (22), 8669-8676.

26. Page, K. A.; Adachi, K. Polymer 2006, 47, (18), 6406-6413.

27. www.scprod.com Southern Clay Products, Inc.

28. Havriliak.S; Negami, S. Polymer 1967, 8, (4), 161-\&.

29. Calvet, R. Clay Clay Min. 1975, 23, (4), 257-265.

30. Kramarenko, V. Y.; Ezquerra, T. A.; Sics, I.; Balta-Calleja, F. J.; Privalko, V. P. J. Chem. Phys. 2000, 113, (1), 447-452.

31. Psarras, G. C.; Gatos, K. G.; Karger-Kocsis, J. J. Appl. Polym. Sci. 2007, 106, (2), 1405-1411.

32. Tsangaris, G. M.; Psarras, G. C.; Kouloumbi, N. J. Mater. Sci. 1998, 33, (8), 2027-2037.

33. Lee, H. K.; Pejanovic, S.; Mondragon, I.; Mijovic, J. Polymer 2007, 48, (25), 7345-7355.

34. Kalgaonkar, R. A.; Jog, J. P. J. Polym. Sci. Pt. B-Polym. Phys. 2008, 46, (23), 2539-2555.

35. Schonhals, A.; Goering, H.; Schick, C.; Frick, B.; Zorn, R. Colloid Polym. Sci. 2004, 282, (8), 882-891.

36. Psarras, G. C.; Gatos, K. G.; Karahaliou, P. K.; Georga, S. N.; Krontiras, C. A.; Karger-Kocsis, J. Express Polym. Lett. 2007, 1, (12), 837-845.

37. Scheidler, P.; Kob, W.; Binder, K. J. Phys. Chem. B 2004, 108, (21), 6673-6686.

38. Fragiadakis, D.; Pissis, P. Journal of Non-Crystalline Solids 2006, 353, 43444352.

39. Hedvig, P., Dielectric Spectroscopy of Polymers. Bristol, 1977.

40. Fritzsche, J.; Das, A.; Jurk, R.; Stokelhuber, K. W.; Heinrich, G.; Kluppel, M. Express Polym. Lett. 2008, 2, (5), 373-381.

41. George, S.; Varghese, K. T.; Thomas, S. J. Appl. Polym. Sci. 1999, 73, (2), $255-$ 270.

42. Abd-El-Messieh, S. L.; Abd-El-Nour, K. N. J. Appl. Polym. Sci. 2003, 88, (7), 1613-1621. 